\title{
SIX WORKS OF ART AND THEIR SIGNIFICANCE FOR THE FUTURE OF COMPUTING
}

\author{
Colin Beardon \\ Exeter School of Arts \& Design \\ University of Plymouth \\ $U K$
}

\begin{abstract}
This article looks towards our material cultures, which are traditionally seen as a source of wisdom sustained across generations, to see whether their study might provide a complementary perspective on current trends and issues within computing. It contains three case studies. The first is concerned with the early history of computing and relates this to works by Roman de la Rose, Caravaggio and Thomas Wright. The second concerns the speed of technological change and this is considered in the light of a work by Turner. The third concerns graphical representation systems and refers to works by Vermeer, Picasso, Estes and others.
\end{abstract}

\section{INTRODUCTION}

There are aspects of the relationship between technologies and their social environment that are so large that it is difficult, if not impossible, to comprehend them from close range. The pursuit of important contemporary issues from within the contemporary intellectual paradigm has some limitations. This article looks towards our material cultures, which are traditionally seen as a source whereby wisdom is sustained across generations, to see whether such a study might provide a complementary perspective on current trends and issues within computing.

In particular I will primarily look at the work of six artists, concentrating upon those aspects which seem to reflect interestingly on issues in the development of computing. In doing this I am not claiming that these artists necessarily anticipated the technologies we have today, or even the specific issues that these technologies have raised. It may be that the artists deliberately touched upon issues that are of such a general nature that we see manifestations of them today, in our very different cultural setting; or it may be that my response to them is purely subjective and that I derive meaning from their work that was in no way intended by the artists themselves. It is not important to me to resolve this issue, I merely want to see whether the contemplation of such works in today's context helps by throwing new light on some issues in the field of computers and society. 
If prior justification for this enquiry is necessary, then it may be found in the adage that art is truth without facts.

To some people, the idea that there can be a truth that is not symbolically represented comes as a shock. Truth, many have come to believe, is always expressed in language - as sentences, statements, propositions, or whatever.

Without entering into a full-scale philosophical discussion, let us observe that, in English, the words 'true' and 'trust' have a common origin. More than that, we can see vestiges of an earlier concept of 'truth' that was closely related to 'trust' in such sentences as 'Peter was a true friend'. In this case we are not simply asserting that 'Peter was a friend' is a true statement (though this may be inferred). Rather, we are asserting that Peter was a particularly clear example of a friend, or that he conformed to our idea of the ideal friend. This would not have been an instantaneous or quick judgement, but something that could only be deduced over time and through experience over a wide range of situations. The real meaning of this sentence is, we could trust Peter to act like a friend is expected to act. That, in this example, is what we mean when we use the adjective 'true'.

We can find even clearer examples of this different concept of 'truth' in the use of the word 'true' within various craft-based activities. A carpenter will look along a piece of timber to see if it is 'true' (i.e. straight) and a stone mason will describe a rectangular building block, or a wall built of them, as 'true' (i.e. consisting of planes at right angles). The idea of 'fitness to purpose' comes in here, and also that of reliability over time. Through examples such as these we can see that we retain a concept of truth that is based upon reliability and relevance over time or, to put it simply, as based upon 'trust'.

In this context, it is interesting to note that one of the major problems with electronic communications today is that, while we can exchange information practically without limit, we lack the ability to establish any concept of trust (at least within a commensurate time scale). Hence we do not know the status of information we may come across, we do not know if our communications are secure, we do not know anything about the person we may be dealing with or sending money to. We are at the stage of Marco Polo, making journeys to strange places, not knowing the customs, whom to trust and who may be out to deceive us.

The attachment to a purely symbolic representation for 'truth' has led us to the concept of 'information' which, as Machlup and Mansfield (1983) among others have pointed out, is a particularly ill-defined concept. Many people today would find it very difficult to say what 'information' really is (from my own experience, I know that this even applies to people who have the term 'Information' in their job title). People also have difficulty distinguishing 'truth' from 'information' and I would suggest that part of the reason for this is that they have lost the aspect of 'trust' that should be inherent in 'truth'. It may therefore be relevant to suggest that, information is knowledge without trust. 
So, the idea that there can be knowledge that is enduring but not in symbolic form leads us to consider the possible role for our (diverse) material cultures. We know that in many societies that differ from ours, cultural artefacts play a significant role as reference points when discussing significant contemporary questions. In such societies, there is often no concept of 'art' such as we have in many western societies today. Cultural artefacts in those societies have an accepted function, while in western societies we have detached them from such a function and created a new, historical and economic context within which they operate (i.e. they are put in galleries for detached study, and exchanged as prized possessions). This can be seen as part of the process of producing 'information' - i.e. cutting knowledge from its longer-term cultural context within which it had become trusted, and hence 'true'.

It is within this intellectual framework that I would now like to approach some works of art and ask of them what they may have to say that is of relevance to the questions of 'computers and networks in the age of globalisation'.

\section{CARAVAGGIO, JOSEPH WRIGHT AND THE FIRST CALCULATING MACHINES}

Let us start at the very beginning of mechanical calculation which, as far as our knowledge is concerned, is the year 1623. It was in this year that Willhelm Schickard built the earliest known calculator, a machine capable of addition, subtraction, multiplication and division - a machine that was unfortunately destroyed in a fire during Schickard's lifetime.

1623 was also the year that Blaise Pascal was born. Pascal is one of the founders of modern probability theory and was the first person to use air pumps to demonstrate the existence of air-pressure and vacuums. At the age of nineteen he developed a calculating machine which became known as La Pascaline. It could only perform addition and subtraction but its 'user interface', as we might say, was an improvement over Schickard's design having eight dials each of which could be manipulated by a stylus. Pascal manufactured over fifty of these machines and they were a great novelty in a society that was fascinated by mechanical dolls and cabinets of curiosities, but once the novelty value had worn off little commercial interest was shown in them.

As far as we know, the production of mechanical calculators was not commercially viable until 1820 when Charles Xavier Thomas de Colmar began to manufacture and sell them from his business in Paris. By 1876 he had produced more than 1200 machines of a design that had changed little since La Pascaline. In fact, companies in the US and Europe were still manufacturing machines to a similar design as late as 1920 . 
The very brief account of the early history of calculating machines raises an important theoretical issue. Why did it take from 1623 until 1820 (that is 197 years) for the existence of the knowledge of how to build a mechanical calculator to lead to the device being purposefully used within society? We are often led to believe that the existence of scientific and technical knowledge is itself a driving force of change, but how would such a determinist explain this delay? There was clearly something other than a simple cause involved.

The image in Figure 1 was produced around 1500 in Flanders and is entitled, Dame Nature gives her orders to Genius. In this view of the world, Nature communicates with human understanding through signs. Natural phenomena, like the arrangement of stars, the features of the landscape or even the entrails of animals, can be read like we read a book. Nature is the author and Science awaits her every pronouncement.

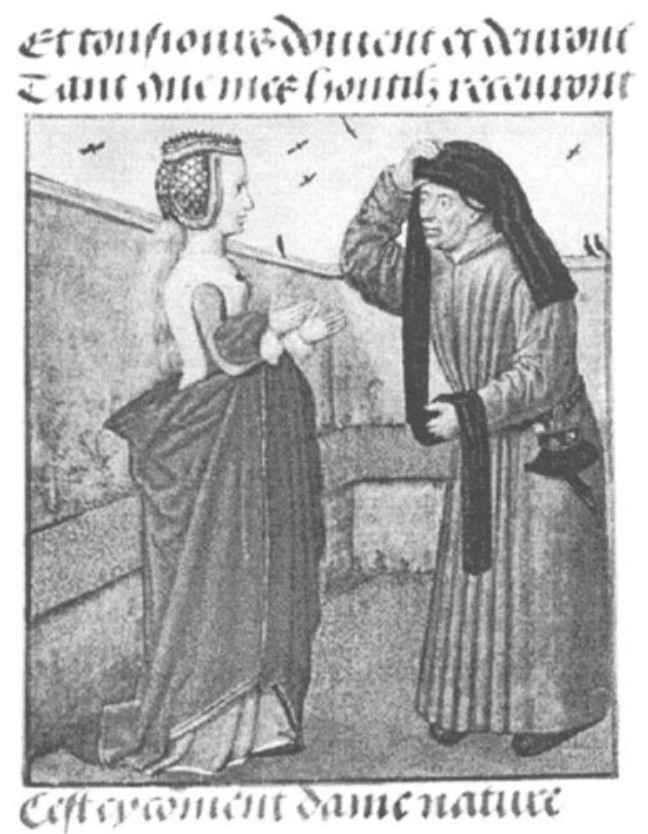

Figure 1. Dame Nature gives her orders to Genius. Roman de la Rose, Flemish, C1500. By permission of the British Library (Ms No. Harley 4425 fl16)

By 1820 the culture in Western Europe had changed. Nature was considered to be repetitive and consistent, and changes in natural phenomena were understood as having causal antecedents rather than symbolic meaning. Furthermore, because Nature was now seen as uniform, she would gradually 
yield her secrets to the ever more effective processes of science. By 1820 , Nature was in the process of being dominated by Science.

Two famous paintings illustrate this difference very well. Figure 2 is The Incredulity of Saint Thomas painted by Caravaggio about twenty years before Schickard built his calculator. Figure 3 is An Experiment on a Bird in the Air Pump painted by Joseph Wright of Derby some forty years before Thomas de Colmar started to sell calculators. I want to use these two images to illustrate some important changes in west European culture over these two centuries.

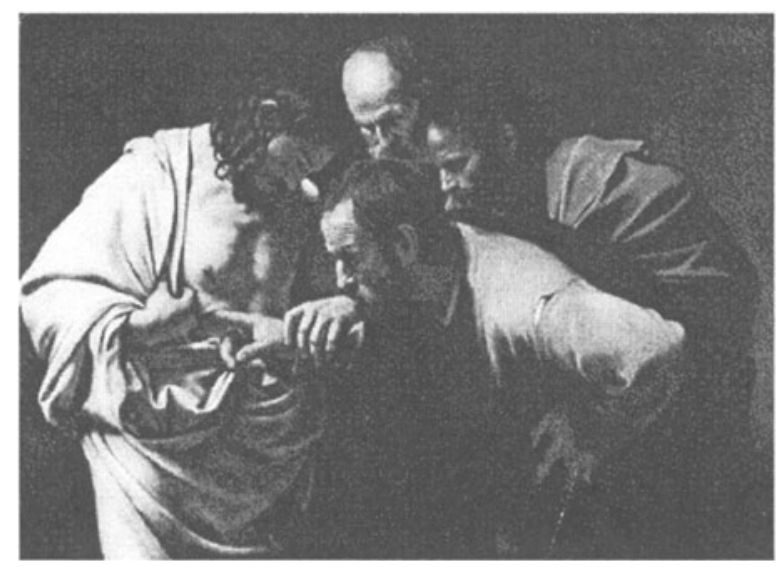

Figure 2. The Incredulity of Saint Thomas. Caravaggio 1601-2. Neues Palais, Potsdam

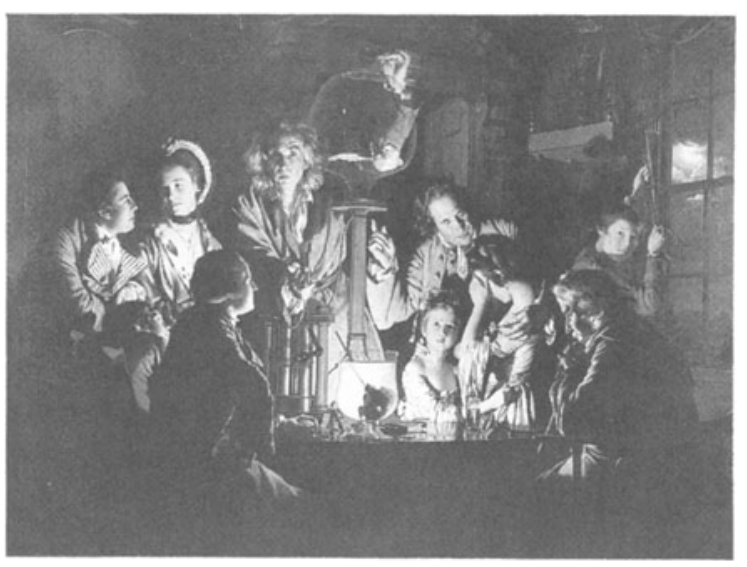

Figure 3. An Experiment on a Bird in the Air Pump. Joseph Wright of Derby, 1734-1797. National Gallery, London 
The two paintings have some obvious similarities. Both depict their scene in a natural manner - there are no halos, no angels, nor any text or overt symbols. Both use dramatic lighting to highlight their subject reducing the background to relative insignificance. Both paintings depict an event, in each case an experiment, through which they refer to the acquisition of knowledge and its implications.

But there are important differences. In the Caravaggio, St. Thomas is perplexed, and the origin of his confusion lies in his attempt to reconcile the two differing views of natural phenomena described above. If Nature conveys meaning through natural events as symbols, then the wound is not a wound as we might understand it in medical terms today, but it is a sign. On the other hand, if Nature is uniform, then the wound must be like any other wound and the person bearing it should behave like any other person in the same circumstances. The naturalness of the setting makes this a very 'modern' painting but this enhances the feeling of incredulity by favouring a modern scientific approach.

Look also at the relationship of the knowledge gained to the person who has gained it. St. Thomas' experiment is very personal and is strictly nonrepeatable. You could not carry out this experiment on any individual at any time. Both subject and object are crucially bound up in a very particular event of knowing and from that act of knowing (or 'believing' as we would express it today) a whole way of life will follow. For St. Thomas, the implications of believing are indivisibly both epistemological and ethical.

Contrast this to the Experiment... (Fig. 3) which was painted in a later time when knowledge was seen as completely objective. The experiment that forms the subject of the painting is a standard, repeatable demonstration that was used to disseminate scientific knowledge. The outcome is predictable in that it is devised so that all the participants will leave the scene knowing the same piece of knowledge. But there is an element of uncertainty: a white cockatoo has been placed in a glass jar in which a vacuum has been created using an air pump (n.b. as first demonstrated by Pascal) and this has resulted in the bird falling unconscious. The future life of the bird depends upon the action of the demonstrator. If he releases his grip on the rubber tube, air will flow back into the jar and the bird will revive; if he does not, then the bird will die. Nature has become totally subordinate to the progress of human knowledge. The primary event is now the scientific demonstration and morality has become a separate and secondary reaction - not connected to the knowledge itself, but a subjective response to it. As a result we can see a wide diversity of ethical responses written on the faces of the audience.

When Pascal tried to sell his calculator as a useful device, the society in which he lived could only categorise the machine as a curiosity. Knowledge had yet to become objectified in the way represented in Figure 3 and the relationship between an action and the person who acts was still intimate. This was the age when one's reputation, one's Name, was all-important as a guarantee of reliability, trust and truth. There were many social actions (e.g. 
promises, oaths, signatures and handshakes) that were ritualised but important signifiers in social life. Consequently, the adding up of a column of numbers was an action bound to the person who performed it and their reputation. Calculation was:

"Allied with the higher mental faculties of speculative reason and moral judgement [and] was remote from the realm of menial labour, of the automatic and the habitual." (Daston 1994)

Before it could become legitimate to mechanise and merchandise arithmetic, it was necessary for it to become objectified and thus separated from the human who performed it. Only then could mechanical calculation, or rather the act of calculating using a mechanical device, be accepted by society.

"Whereas the adding machines designed by Pascal had entertained heads of state ... the general-purpose calculators of the early nineteenth century were viewed with increasing interest by heads of insurance companies." (Merzbach 1977)

The mere existence of the 197 year gap between prototype and commercial product shows that there is nothing inevitable about the forward march of computing. As we have seen, it can stop for two centuries until the right cultural conditions exist. This study of just two paintings throws some light on the mechanisms whereby a technology is mediated by a society. It reminds us, for example, that using a computer is an action that is performed by a subject who cannot avoid certain social responsibilities. It reminds us that knowledge must have a knowing subject (unlike 'information') and that the description of the computer as a processor of information is an attempt to separate knowledge from any particular subject.

\section{J.M.W. TURNER AND THE INDUSTRIAL AND INFORMATION REVOLUTIONS}

"The length of our lives, so far as regard the power of acquiring information and disseminating power, will be doubled, and we may be justified in looking for the arrival of a time when the whole world will have become one great family, speaking one language, governed in unity by like laws, and adoring one God." (Francis 1851)

Reverend Francis was, of course, describing the impact of railways, not the impact of computers, but given a slightly less archaic form of expression (and more hyperbole) and the same passage might be used today to express some people's reaction to the speed of development of computer and communications technologies.

In 1844, J.M.W. Turner first exhibited a painting called simply, Rain, Steam, Speed (Figure 4) which is quite clearly the artist's reaction to the new presence of railways in Britain. Between about 1830 and 1845 a country that had relied upon horse drawn coaches for long distance travel was almost completely connected by railways. The country was gripped in a 
'railway fever' as this potent symbol of industrialisation made gargantuan strides across the countryside. The painting does not attempt to document in any detail the technology itself, its extent, nor any of the complex events that surrounded it (such as building the railway, or the emotive scenes that take place at railway stations), but is almost obsessed with the pure sensation of speed, heightened by the effect of rain.

What does this image have to tell us about the future of computing today? In some simple ways we can see it as a kind of metaphor for the 'Information Age'. We have an awesome and powerful technology leading the way, pulling along carriages full of people who merely follow in its wake. It is running along fixed tracks and so has very little flexibility, yet the tracks were laid by somebody. But more important than all of these is that sensation of speed itself. This is the core of the painting and it is a sensation that we have, or at least are told that we have, today.

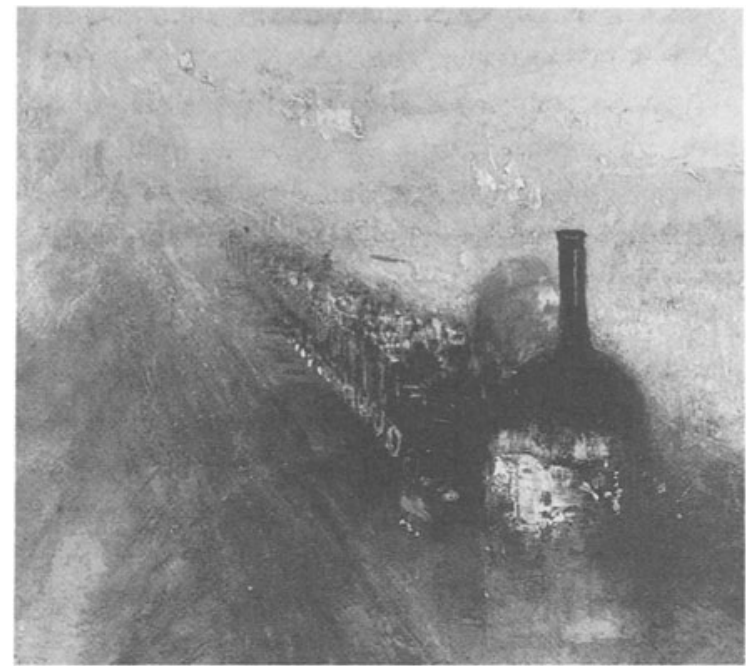

Figure 4. Rain, Steam, Speed (detail) J.M.W. Turner. National Gallery, London

Prior to the advent of passenger trains, journeys could be very slow. In 1841 the fastest journey from London to Bristol by road (about $180 \mathrm{Kms}$ ) took over twelve hours. When the first passenger train was introduced in that year the regular time for the same journey was four hours and in speed trials locomotives had reached $135 \mathrm{Km} /$ hour. But for Turner the main impact was the sensations experienced by ordinary people, like the effect of one locomotive passing another going in the opposite direction (a relative speed in excess of $150 \mathrm{Km} /$ hour). Their ability to overcome the most adverse weather conditions only heightened the excitement and awe (Gage 1972).

We think, today, that the Information Revolution is going faster and faster. We are told that the power of computers is increasing exponentially and that 
the generation of knowledge (as measured by the number of published papers) is faster than ever before. By comparison, the age of steam seems only vaguely comparable: we believe that we are both travelling and accelerating at a far greater rate than our Victorian predecessors. But can we trust these sensations?

To gain a sense of perspective, let us compare the development of the railways as the infrastructure of industrialisation in Britain up to 1850, with the development of computer networks as the infrastructure of the information age up to the present. Both, engendered a great sensation of speed but what was the reality?

Though the first steam locomotive was built in 1804, it was not until 1825 that the first commercial service using a steam locomotive was opened in the North-East of England and it was 1836 before the train network had reached London. The first popular usage of trains, the excursion to the seaside, commenced in 1844 . And by 1850 the national railway network was more or less complete. From then until its high point in the early part of the 20th Century, the usage of all forms of public transport grew dramatically. It has been estimated that between 1830 and 1910 there was an increase from an average of 4 journeys on public transport per head of population per year, to 120 (Simmons 1991).

The various private railway companies operated different sized equipment up until 1846, when the gauge was standardised. The requirement to produce train timetables led to a different kind of standardisation: the replacement of local by standard time throughout Britain. The social impact of railways went far beyond the ability to travel and the introduction of national standards. Not only were thousands of new jobs created in the railway industries but there were also significant advances in engineering (through the building of major new bridges and tunnels) and new forms of architecture (railway stations, hotels, etc.). The advent of the railway had a major effect on all forms of communications (e.g. the postal service became much faster and cheaper, the telegraph was developed, and newspaper distribution became easier). It also brought about large scale transformations of social life (e.g. excursions to the seaside, the building of many hotels, the development of steamships which encouraged overseas travel, etc.).

The history of computing and communications technology has many similarities to the history of railways. The accolade of 'first computer' is the subject of much debate but, for the purposes of comparison I will place it as around 1945. The first commercially usable device was the UNIVAC 1 in 1951. During the next thirty years computers were used almost entirely within large organisations for data processing, up until the advent of personal computers around 1980. While networking has been available for many years, it did not really become popular until the Internet and its various standard protocols, which stabilised around 1995.

Let us simplify these events and tabulate them. 


\begin{tabular}{|l|rr}
\hline Event & 1804 & 1945 \\
Prototype & 0 & 0 \\
Commercial use & +21 & +6 \\
Popular impact & +40 & +35 \\
Standardisation & +42 & +50 \\
Network complete & +46 & +50 \\
\hline
\end{tabular}

This table is clearly schematic rather than precise and there could be much dispute about precise dates and claims and counter-claims to be first with this or that development. Any such disputes miss the main point which, I believe, is irrefutable. The development of computer and communications technology, as far as the vast majority of the population is concerned, is going no faster than the industrial revolution was going for our Victorian forebears. Furthermore, when we consider the breadth of the impact of railways, with their associated employment, their developments of engineering and architectural constructions, their effect on communications and on social life, then we may seriously wonder whether the information revolution is as significant to us as the railway was to them. Despite our impressions of great speed, we are really progressing no more quickly nor more profoundly.

Finally, we believe that our technology, Information and Communications Technology, is different because its impact is global rather than national. This may be the case, though we must also remember that railways were exported from the major industrialised countries and soon made a major impact of countries around the world, and that well over $90 \%$ of the world's population have never used any kind of computer technology (though, of course, they may be affected by it). My major point, however, is that our perception of globalisation may be as equally defective as our perception of speed.

\section{THE AVANT GARDE, COMPUTER GRAPHICS AND COLLAGE}

For the first forty years of the development of computing nearly all machines operated with input and output that represented the real world through symbols, usually encoded in a standard format such as ASCII. It is only relatively recently that we have had easy access to graphical interfaces with their ability to display and manipulate high-quality colour pictures, sounds, moving images, etc. The fundamental issue raised by this new multimedia context of computing is that of semantics. How has the way that computing objects generate possible meanings changed with the advent of multimedia?

There is a famous saying that "a picture is worth a thousand words". This is literally true in terms of binary storage: the informational content of the text 
contained in a typical article is about 30 thousand bytes, whereas each image in this article could occupy up to $20 \mathrm{Mb}$, or over six hundred times the size of the text. Similarly, digitally recorded spoken voice would be hundreds, if not thousands, of times larger than the same words stored as text. Such massive increases in bandwidth represent a qualitative change in the capacity of computers to convey meaning and they require that we develop new theoretical models for understanding computing output based upon the richer medium it now provides.

When considering the role of pictures as a medium, there is a commonly held view that there exists a single 'realistic' or 'natural' image that can be produced from any scene and that this is more or less equivalent to what we would expect to be produced by a camera placed at the spot where the artist was standing. Devices have been used throughout history to enable drawings constructed in this manner, and they have sometimes been recorded by artists themselves (e.g. Dürer's The painter studying the laws of foreshortening, 1525). The principle is also very evident in the work of Vermeer. So accurate is the rendition in Vermeer's The Music Lesson that it has been possible to deconstruct the image and recreate an accurate 3dimensional model of the depicted scene (Dubery \& Willats 1983 p.78). It now seems clear that Vermeer worked directly with some kind of optical device (such as a camera obscura). Evidence for this comes from the fact that his paintings have a constructed focal depth (i.e. objects beyond this range are painted slightly 'out of focus').

The artistic movement known as 'photorealism' might be assumed to embody this supposedly 'real' approach to visual representation, but it was a deliberate attempt by artists to make paintings that are, in some sense, more photographic than the photograph itself (e.g. Richard Estes Bus Reflections 1972). A photograph captures a frozen moment in time, lasting hundredths of a second, but a 'photorealist' painting may differ slightly from the photographic image in order to enhance the quality of being a fragment from some narrative.

The Cubists were the first to reject the projection of light onto a flat plane as the main organisational principle and they developed several techniques of fragmentation and reconstruction. In 'projected' paintings the boundaries of a particular object is often lost in shadows, so that it is impossible to say by looking at the detail where the boundary between a person and the background occurs. Manet was probably the first to adopt a different approach by introducing the technique of depicting objects in terms of a flat and clearly delineated area (e.g. L'Olympia 1863) (Richardson 1971).

Cubism was interested in the question of how a three dimensional object might be represented on a two dimensional surface. Given that we visually perceive objects by means of active exploration, with our eyes moving over the surface of the object and our bodies moving in relation to the object, the attempt to record an object by means of a documented journey can be seen as more real and true to nature. In some works (for example, Picasso's Femme á la mandoline 'Fanny Tellier' 1910) the object is not flattened onto 
a two-dimensional plane but represented through fragments from a visual journey in three-dimensions, organised into a unified whole. While the cubists generally took an object or collection of objects that was static over time (e.g. a still life) and recorded a viewer's journey in space around the object, the Futurists performed a similar deconstruction and reconstruction but kept the viewer still in front of an object that moved over time, showing fragments of the same object at different time periods within the same picture.

The group of artists known as the Purists - Ozenfant, Jeanneret (later known as Le Corbusier) and Léger - developed the idea of fragmentation within the context of mechanised production. The idea of fragmentation remains a strong principle in paintings such as Jeanneret's Still-life with Pile of Plates.

"Although the objects are schematized and flattened out, each object remains self-contained and unambiguously depicted: objects are clearly differentiated from each other as well as the space they inhabit." (Batchelor 1993 p.22)

Others were interested in the fragmentation of the process of artistic production. While Kandinsky was later to write about "an art of replaceable parts" (Richardson 1971 p. 158), it was the Russian Constructivists who were the first to combine the fragmentation of cubism with the ideas of Frederick Taylor on the division of labour,

"... artistic construction .. was treated as a system that could be broken down into component parts. Its elements could be analysed, dissected and put back together again." (Fer 1993 p.108)

Though Jeanneret was concerned that the division of labour might lead to the loss of craft skills, he thought that they would be compensated by feelings of solidarity and pride in perfection.

The combination of fragmentation with the drive towards greater efficiency in production (the crucial link between 'modern art' and 'modernisation') led the Purists towards the elimination of all forms of decoration and a drive towards mathematical simplicity (which was later to become a founding principle of the Bauhaus). In the works of Léger (e.g. Le Grand Déjeuner 1921) we see the "key features of modernization - repetition, standardization, rationalization, efficiency, accuracy, precision, timekeeping." (Fer 1993 p. 140).

The task of a work of art, in their aesthetic view, was to strip away culturally bound 'secondary sensations' and identify what is universal and common across all cultures.

"In summary, a work of art should induce the sensation of mathematical order, and the means of inducing this mathematical order should be sought among universal means." (Jeanneret \& Ozenfant 1920). 
Their aesthetic theory was based upon a simple universal belief,

"Man is a geometric animal, animated by a geometric spirit." (Ozenfant \& Jeanneret 1918)

One can detect the effect of this kind of aesthetics on today's computer graphics, founded as they so often are, on the application of mathematics to a constructive solid geometry (Foley et al 1990). More recently, however, we have seen the possibilities of using two-dimensional representational techniques, many made popular by the application Adobe Photoshop ${ }^{\mathrm{TM}}$. This allows for the more explicit use of artistic fragmentation, as we find made more explicit in collage.

The origin of collage is often taken to be Still Life with Chair Caning (1912) in which Picasso chose to represent an oil cloth, not by trying to capture a perspectival representation using paint, but rather by introducing a real piece of oil cloth into his painting, thus posing the question of whether we are to say that this painting is less 'real' because it uses a real oil-cloth?

For many collage (and montage) is "the single most revolutionary formal innovation in artistic representation to occur in our century" (Ulman 1985). Certainly, for artists such as John Heartfield, the ability to work with official material in such a way as to completely subvert its meaning was important both relatively and politically. More recently, feminist artists have found that the appropriation and re-use of imagery can create an interpretative space.

Yet there is only recently emerging an aesthetic representational form within computer graphics that builds upon collage/montage. The dominance of a slick, modernist avant garde approach that is strictly functional and geometric is still noticeable.

\section{SUMMARY}

This paper is an attempt to make a methodological statement: that when considering contemporary technologies and their relationship to society we should acknowledge our longer-term historical reference points - for example, our material culture. I have tried to show this by relating how, for me, contemplating certain works of art while, at the same time, thinking about certain issues of concern to 'computers and networks in the age of globalisation' led to some thought processes that maybe I would not have previously had.

I certainly feel that stages in the history of computing cannot be fully understood without understanding the very different cultural conditions which applied at the time, and that our pictorial heritage is an important indicator of this. I also think that the broader sweep of technology-related 
historical change (e.g. an 'information age') is similarly better understood by an appreciation of the cultural conditions of different periods that had similar experiences.

These cases are slightly different from the third, that of computer graphics. Here, I would argue that the future direction of a technology might be well informed by an appreciation of some of the more fundamental concerns of the artistic world. At a technical level, computers are a product of late modernism and remain rooted in this ideology (and very successfully so). But they are now becoming so complex that the meanings that they produce can no longer be understood within this paradigm - their output is more closely akin to films and multimedia spectacles of all kinds. The meanings behind the range of representational systems that have been considered then become a real issue for the future of computing.

This paper is, in many respects, an initial exploration into this new field. My hope is that it is sufficiently convincing that others will see possibilities here and will follow with more detailed and substantial contributions in the future.

\section{REFERENCES}

Batchelor, D. (1993) 'This Liberty and this Order': Art in France after the First World War. In: Fer, B., Batchelor, D. \& Wood, P. Realism, Rationalism, Surrealism: Art between the Wars. Yale UP, London, pp 2-86.

Daston, L. (1994) Enlightenment Calculations. Critical Inquiry 21(1).

Excerpt on http://www2.uchicago.edu/jnl-crit-inq/v21/v21 n1.daston.html

Dubery, F. \& Willats, J. (1983) Perspective and other drawing systems. The Herbert Press, London.

Fer, B. (1993) The Language of Construction. In: Fer, B., Batchelor, D. \& Wood, P. Realism, Rationalism, Surrealism: Art between the Wars. Yale UP, London, pp 87-169.

Foley, J., van Dam, A., Feiner, S. and Hughes, J. (1990) Computer graphics: principles and practice. Second edition. Addison-Wesley, Reading, Mass.

Francis, J. (1851) A History of the English Railways. It's Social Relations and Revelations. Newton Abbott, UK.

Gage, John (1972) Turner: Rain, Steam \& Speed. London, Allen Lane The Penguin Press.

Jeanneret, C.-E. \& Ozenfant, A. (1920) Purism. L'Esprit Nouveau, pp. 59, 61.

Machlup, F. \& Mansfield, U. (eds.) (1983) The study of information. John Wiley \& Sons, New York.

Merzbach, U. (1977) Georg Scheutz and the first Printing Calculator. Smithsonian Studies in History and Technology, No. 36. Smithsonian Institute Press, Washington.

Ozenfant, A. \& Jeanneret, C.-E. (1918) Aprés le Cubisme. Paris. 
Richardson, J. A. (1971) Modern Art \& Scientific Thought. University of Illinois Press, Urbana, Ill.

Simmons, J. (1991) The Victorian Railway. Thames \& Hudson, London.

Ulmer, G. (1985) The object of post-criticism. In Foster, H. (ed.) Postmodern culture. Pluto Press, London, pp. 83-110. 